\title{
Concepções Pessoais de Competência e Rendimento Acadêmico: Estudo Longitudinal no Ensino Secundário Português*
}

\author{
Joana Stocker ${ }^{1, * * * *} \&$ Luisa Faria ${ }^{2}$ \\ 'Zayed University, Dubai, Emirados Árabes Unidos \\ ${ }^{2}$ Faculdade de Psicologia e de Ciências da Educação da Universidade do Porto, Porto, Portugal
}

\begin{abstract}
RESUMO - As concepções pessoais de competência (CPC) influenciam o rendimento escolar e/ou o contrário? Com base nesse questionamento, neste estudo pretendeu-se analisar a relação entre CPC (concepções pessoais de inteligência, atribuições causais, autoconceito, autoeficácia e competência emocional) e o rendimento escolar ao longo do ensino secundário, especificamente em Português. Com esse objetivo, administrou-se o Questionário Compósito de Competência Percebida (162 itens) para 433 alunos, entre os quais estão 52,8\% de meninas com idade entre 14 e 18 anos $(M=15,3$; $D P=0,63)$, num desenho longitudinal com três momentos separados por um ano de intervalo. Globalmente, os resultados revelaram relações recíprocas: o rendimento de um ano influenciou as CPC no ano seguinte e o autoconceito e a autoeficácia foram os principais preditores do rendimento escolar.
\end{abstract}

PALAVRAS-CHAVE: competência percebida, longitudinal, rendimento escolar, ensino secundário

\section{Personal Conceptions of Competence and Academic Achievement: Longitudinal Study in Portuguese Secondary School}

\begin{abstract}
Do personal conceptions of competence (PCC) influence academic achievement and/or academic achievement influences PCC? In this context we sought to examine the relationship between PCC (personal conceptions of intelligence, causal attributions, self-concept, self-efficacy and emotional competence) and academic achievement throughout secondary school in Portugal. With this aim, the Composite Questionnaire of Perceived Competence (162 items) was administered to a sample of 433 students, $52.8 \%$ girls, aged between 14 and 18 years $(M=15.3 ; S D=.63)$, in a three-wave longitudinal design separated by a gap of one year. Overall, the results evidenced reciprocal relationships: the achievement in the first year influenced PCC in the following year and self-concept and self-efficacy were the main predictors of academic achievement.

KEYWORDS: perceived competence, longitudinal, academic achievement, secondary school
\end{abstract}

Neste trabalho haverá o retorno à discussão da seguinte questão de investigação, a qual é largamente discutida na literatura científica que trata sobre competência e desempenho (Bandura, 1977; Marsh, Byrne, \& Yeung, 1999; Marsh \& Martin, 2011; Pajares \& Schunk, 2001): serão as concepções de competência individual que influenciam o rendimento acadêmico ou o inverso, em que o rendimento é um influenciador nas concepções que os indivíduos constroem acerca da sua competência em contextos de realização escolar? Baseado nesse questionamento, será analisado o impacto das concepções pessoais de competência (CPC) nos resultados escolares de alunos do ensino secundário e, simultaneamente, o impacto dos resultados escolares nas CPC dos alunos.

O Modelo das Concepções Pessoais de Competência, já investigado no contexto escolar português no quadro de vários estudos nos últimos anos (Pina Neves \& Faria, 2005; Stocker, Pina Neves, \& Faria, 2011; Stocker \& Faria, 2012), analisa e explica as CPC a partir da noção de teorias pessoais implícitas a que cada um recorre para compreender, explicar e prever a ocorrência de sucesso ou de fracasso nos contextos de realização, especificamente no contexto acadêmico, o que influencia a motivação para a competência e, em última instância, a própria realização. Enquanto constructo global e

\footnotetext{
* Esta investigação foi financiada pela Fundação para a Ciência e a Tecnologia no âmbito de uma bolsa de doutoramento, concedida a Joana Stocker (SFRH/BD/60225/2009), realizada na Faculdade de Psicologia e de Ciências da Educação da Universidade do Porto, Portugal.

**E-mail: joana.stocker@zu.ac.ae
} 
integrador, as CPC integram variáveis relativas às percepções de competência pessoal dos alunos, entre as quais estão: (a) as concepções pessoais de inteligência (CPI) (Dweck, 1996) - crenças acerca da natureza desenvolvimental da competência intelectual, com probabilidade de serem mais estáticas (inatas) ou dinâmicas (passíveis de desenvolvimento); (b) as atribuições e dimensões causais (Weiner, 1985) - explicações causais dos acontecimentos escolares, variando quanto ao locus (interno/ externo), estabilidade (estável/instável) e controlabilidade (controlável/ incontrolável); (c) o autoconceito acadêmico (Marsh et al. 1999) - percepções acerca das competências acadêmicas individuais: autoconceito verbal, Matemática e assuntos escolares; (d) a autoeficácia acadêmica (Bandura, 1977) - expectativas de eficácia pessoal quanto às realizações escolares: autoeficácia em Português, Matemática e escolar geral; (e) competência emocional (Mayer \& Salovey, 1997) - capacidade para reconhecer e compreender emoções em si mesmo e nos outros (percepção emocional) e de expressar e regular emoções (expressão emocional e capacidade para lidar com a emoção, respectivamente).

Ora, vários estudos comprovaram que as variáveis integrantes do modelo das CPC estabelecem relações entre si, bem como com o rendimento acadêmico (Bandura, 1977; Mayer \& Salovey, 1997; Pina Neves \& Faria, 2005; Weiner, 1985). Neste estudo será retomado o reciprocal effects model (Byrne, 1984; Marsh et al., 1999; Marsh \& Martin, 2011), que propõe a existência de efeitos recíprocos entre o autoconceito e o rendimento. Além disso, será discutido sobre o modelo das CPC, propondo relações entre os seus constructos (relações interconstructos), como o rendimento acadêmico.

\section{RELAÇÕES INTERCONSTRUCTOS DAS CPC}

No que diz respeito às relações entre as variáveis do modelo das CPC, este estudo propõe o tratamento das dimensões das CPI e da competência emocional como constructos organizadores das concepções de competência, precedendo e influenciando os outros (Dweck, 1996; Pina Neves \& Faria, 2005; Yeager \& Dweck, 2012). Diferentes estudos revelam que o tipo de CPI (estática ou dinâmica) afeta diretamente a construção dos estilos atribucionais e do autoconceito e, indiretamente, tendo o autoconceito como mediador, a autoeficácia. Mais concretamente, alunos com CPI mais dinâmicas tendem a interpretar as causas como mais internas, instáveis e controláveis, dando mais importância ao papel do esforço, o que gera um sentimento de competência (autoconceito positivo) e, através disso, a percepção de que são eficazes, construindo expectativas acadêmicas mais positivas (Blackwell, Trzesniewski, \& Dweck, 2007; Ferla, Valcke, \& Cai, 2009; Pina Neves \& Faria, 2005; Yeager \& Dweck, 2012).

Embora a relação da competência emocional com outros constructos psicológicos não se encontre amplamente estudada, Mayer e Salovey (1997) apontam para a sua importância na construção de um self positivo, no bemestar psicológico, nas relações sociais, na interpretação de acontecimentos e experiências em diferentes contextos de realização (Brackett, Rivers, \& Salovey, 2011; Gonzaga \& Monteiro, 2011). Assim, o modelo das CPC propõe-se analisar o impacto das dimensões emocionais sobre o autoconceito, autoeficácia e atribuições causais.

Por sua vez, prevê-se que as dimensões do autoconceito influenciem positivamente na formação de expectativas de autoeficácia, sobretudo em domínios de realização afins (Ferla et al., 2009; Pajares \& Schunk, 2001; Pina Neves \& Faria, 2005). Além disso, pode-se falar nas percepções de causalidade, em que os alunos com melhor autoconceito tenderão a formar expectativas acadêmicas mais positivas e percepções com maior internalidade e controlabilidade (Pina Neves \& Faria, 2005; Weiner, 1985). É importante destacar que não há estudos que sustentem previsão fundamentada relativamente à estabilidade.

Na perspetiva atribucional, Weiner (1985) assume que as percepções de causalidade, principalmente a estabilidade, são precursoras da autoeficácia e também uma via para os alunos estimarem a sua eficácia e construírem expectativas. Assim, alunos com tendência a perceber as causas dos acontecimentos acadêmicos como mais internas, estáveis e controláveis, apresentariam expectativas mais positivas (Pina Neves \& Faria, 2005, 2007).

\section{RELAÇÕES ENTRE OS CONSTRUCTOS DAS CPC E O RENDIMENTO}

Baseado no modelo dos efeitos recíprocos, o das CPC propõe relações de impacto recíprocas entre os respectivos constructos e o rendimento escolar. Uma vez que o autoconceito é o único dos impactos do rendimento sobre as variáveis do modelo das CPC que está empiricamente aprofundado, o estudo propõe analisar a influência do autoconceito nos cinco constructos integradores do modelo. Já a relação causal dos constructos psicológicos sobre o rendimento se encontra profusamente estudada, pelo que propomos relações específicas. Assim, as CPI, o autoconceito, a autoeficácia e as atribuições e dimensões causais influenciariam diretamente o rendimento escolar, no sentido em que CPI mais dinâmicas, níveis mais positivos de autoconceito, de autoeficácia acadêmicos e percepções causais mais internas, estáveis e controláveis determinariam positivamente o rendimento (Caprara et al., 2008; Dweck, 
1996; Ferreira et al., 2002; Oliveira \& Soares, 2011; Paiva \& Lourenço, 2011; Pina Neves \& Faria, 2005; Stocker et al., 2011; Yeager \& Dweck, 2012). Estudos revelam resultados controversos quanto à competência emocional (Brackett et al., 2011), os quais ora são significativos (Berger, Alcalay,
Torretti, \& Milicic, 2011) ora não significativos (Ahammed, Abdullah, \& Hassane, 2011; Stocker et al., 2011). Propõese, mesmo assim, que esta competência tenha um impacto positivo no rendimento de forma indireta, através dos outros constructos (Petrides, Frederickson, \& Furnham, 2011).

\section{OBJETIVOS}

Este estudo teve como objetivo principal analisar a relação entre as variáveis do modelo das CPC anteriormente apresentado e o rendimento em Português e Matemática ao longo dos três anos $\left(10^{\circ}, 11^{\circ}\right.$ e $\left.12^{\circ}\right)$ do ensino secundário Português (que correspondem ao $1^{\circ}, 2^{\circ}$ e $3^{\circ}$ anos do ensino médio brasileiro). Mais especificamente, foram contemplados quatro objetivos principais: (a) analisar as relações entre as variáveis psicológicas (relações interconstructos) anteriormente propostas em cada um dos anos em análise; (b) analisar o impacto das variáveis de cada ano letivo sobre o rendimento desse mesmo ano; (c) analisar o impacto do rendimento do ano anterior sobre as variáveis no ano seguinte, ou seja, perceber como as notas do $10^{\circ}$ ano influenciam as variáveis no $11^{\circ}$ ano e como as notas deste ano influenciam as variáveis no $12^{\circ}$ ano; (d) analisar o impacto das variáveis sobre si mesmas ao longo dos três anos, prevendo que as variáveis no $1^{\circ}$ momento tenham impacto positivo e significativo nas respectivas variáveis no $2^{\circ}$ e $3^{\circ}$ momento e que as variáveis do $2^{\circ}$ momento tenham o mesmo tipo de impacto no $3^{\circ}$ momento (Marsh et al., 1999). Apesar de diferentes estudos (Marsh et al., 1999; Marsh \&
Martin, 2011) apontarem para a influência direta do rendimento acadêmico de anos anteriores no rendimento futuro, a presente investigação não contempla esta hipótese de estudo, pois os objetivos principais prendem-se às variáveis das CPC e sua relação com o rendimento.

Para a concretização dos objetivos, foi utilizada uma metodologia longitudinal, com três momentos de observação separados por um ano de intervalo, ou seja, cada momento ocorre um ano após o anterior, de modo a recolher dados no $10^{\circ}$ ano de escolaridade (fase A), no $11^{\circ}$ ano (fase B) e no $12^{\circ}$ ano (fase $\mathrm{C}$ ) dos alunos. A Figura 1 representa a parte estrutural do modelo testado, isto é: as relações interconstructos ao longo das três fases (A, B e C - objetivo [a]), as relações entre as variáveis psicológicas e o rendimento em cada fase (objetivo [b]) e as relações entre o rendimento e as variáveis entre as fases A e B e entre as fases B e C (objetivo [c]). Por motivo de simplificação, as relações de impacto das variáveis psicológicas sobre si mesmas, ao longo dos três anos (objetivo [d]), não foram integradas na Figura 1.

\section{MÉTODO}

\section{Participantes}

Participaram dos três momentos deste estudo 433 alunos do ensino secundário do Porto (Portugal) de diferentes cursos (ciências e tecnologias, ciências socioeconómicas, artes visuais, e línguas e humanidades) de escolas públicas $(54,7 \%)$ e privadas. A maior parte dos alunos era do sexo feminino $(52,8 \%)$ e, no primeiro momento de observação, apresentavam idades entre os 14 e 18 anos $(M=15,3 ; D P=$ 0,63 ). Procurou-se, assim, obter uma amostra representativa de diferentes realidades sociais, culturais e económicas, com uma seleção aleatória das turmas.

\section{Instrumento}

Para avaliar os constructos do modelo das CPC, foi construído o Questionário Compósito de Competência Percebida (QCCP), que resulta da adaptação de cinco instrumentos preexistentes, posteriormente adaptados e integrados neste questionário compósito, com um total de 162 itens: (a) Escala de Concepções Pessoais de Inteligência (ECPI) (Faria, 2006), com duas dimensões: estática e dinâmica (10 itens cada); (b) Questionário de Atribuições e Dimensões Causais (QADC) (Pina Neves \& Faria, 2007), com três dimensões: locus, estabilidade e controlabilidade (16 itens cada); (c) Self-Description Questionnaire III (SDQIII), adaptado por Faria e Fontaine (1992) para avaliar o autoconceito, com três dimensões: autoconceito verbal, Matemática e assuntos escolares (10 itens cada); (d) Escala de Autoeficácia Acadêmica (EAEA) (Pina Neves \& Faria, 2007) com três dimensões: autoeficácia Português (7 itens), Matemática (8 itens) e escolar geral (7 itens); (e) Questionário de Competência Emocional (QCE), adaptado por Faria e Lima Santos (2011), com três dimensões: percepção emocional, expressão emocional e capacidade para lidar com emoção (14 itens cada). No que diz respeito à consistência interna do $\mathrm{QCCP}$, a análise do alpha de Cronbach revela bons valores para todas dimensões, situando-se especificamente entre 0,73 e 0,94 , sendo as dimensões da EAEA e do SDQIII no domínio da Matemática as mais consistentes. 


\section{Procedimentos}

Após a autorização da direção de cada uma das escolas, oficializada através de um documento escrito, foi pedida a autorização dos encarregados de educação dos respectivos alunos numa carta explicativa sobre a temática, os objetivos do estudo e as condições de administração dos questionários e seu caráter confidencial e voluntário. Dessa forma, foi assinando um termo de consentimento livre e esclarecido. A administração do QCCP foi realizada por turma, em tempos letivos e em sala de aula, com a presença do respectivo professor e de um investigador treinado para o efeito, explicando aos alunos os objetivos do estudo, o caráter voluntário da sua participação e a confidencialidade dos dados. O preenchimento do questionário variou entre $20 \mathrm{e}$ 40 minutos. Quanto ao rendimento dos alunos, as escolas facultaram as notas finais dos alunos participantes.

\section{Análise dos Dados}

Como já foi referido, o modelo das CPC proposto neste estudo baseia-se no modelo de efeitos recíprocos, além da análise das respectivas sugestões metodológicas de Byrne (1984) e de Marsh et al. (1999): (a) análise das qualidades psicométricas do(s) instrumento(s) e existência de, pelo menos, três itens por fator; (b) recolha de dados em dois momentos diferentes, no mínimo, e, se possível, em anos de escolaridade diferentes; (c) testar efeitos recíprocos entre as variáveis psicológicas e o rendimento, através de um modelo do tipo full-forward multiwave-multivariable, realizado com recurso à modelação de equações estruturais, modelo causal com variáveis latentes e manifestas e com relações intra e interfases (Marsh et al., 1999).

Relativamente à alínea (a), cada dimensão do QCCP integrou mais de três itens e as suas qualidades psicométricas foram analisadas em estudos anteriores com apoio principalmente em análises fatoriais confirmatórias (AFC), que sugeriram a necessidade de continuação de melhoramento do instrumento, uma vez que alguns dos indicadores de ajustamento global se situaram um pouco abaixo do recomendado $\left(\chi^{2}=6006,9 ; p=0,00 ; g l=2429 ; \chi^{2} /\right.$ $g l=2,47 ; \mathrm{NNFI}=0,92 ; \mathrm{CFI}=0,92 ; \mathrm{RMSEA}=0,04)$. Esses estudos permitiram avaliar o modelo de medida das CPC apresentado neste trabalho apenas com os itens que integraram as AFC anteriores (Maroco, 2010; Marsh et al., 1999). A alínea (b) também foi devidamente cumprida, uma vez que os dados, como já foi referido, foram recolhidos através dos mesmos alunos em três momentos com um ano de intervalo, ou seja, em três anos de escolaridade diferentes. Finalmente, a alínea (c) traduziu-se nas relações de impacto propostas para este modelo e apresentadas nos objetivos deste trabalho (Figura 1). Contudo, devido à complexidade do modelo das $\mathrm{CPC}$ no que concerne ao número de variáveis por fase e de relações intra e interfases, e dessa forma, de parâmetros a estimar, foi necessário realizar as análises de equações estruturais apenas com variáveis manifestas, transformando as variáveis latentes em observáveis, através do somatório dos respectivos itens. Apesar de ser uma abordagem mais limitadora, que pode originar alguma perda de informação (latente), pode-se considerar que essa é a forma mais eficaz e parcimoniosa de cumprir e dar resposta aos objetivos propostos.

Assim, com apoio no software AMOS (versão 19), também com base no mesmo modelo de relações inicial (Figura 1), foram analisados dois modelos diferentes, um para cada tipo de rendimento (Português e Matemática), construídos passo a passo, confirmando a diminuição significativa de $\chi^{2}$ entre os diferentes modelos reespecificados: a) na fase A, por uma questão de parcimónia, foram excluídas, uma a uma, as trajetórias não significativas (Maroco, 2010); b) quando todas as trajetórias da fase A se tornaram significativas, replicouse esse modelo para a fase $\mathrm{B}$, fazendo nova verificação da significância de todas as trajetórias e retirando as não significativas, procedendo da mesma forma na fase $\mathrm{C}$; $\mathrm{c}$ ) em cada uma das fases introduziram-se covariâncias entre os erros de dimensões do mesmo constructo, de modo a contemplar a sua relação; d) foram previstas trajetórias de influência de cada variável psicológica sobre si própria ao longo das três fases (da fase A para a B e para a C, e da fase $\mathrm{B}$ para a $\mathrm{C}$ ); e) relativamente ao rendimento, apesar de não se pretender analisar o impacto sobre si mesmo ao longo do ensino secundário, a possível existência desta relação foi contemplada através da correlação dos respectivos erros entre as três fases, de modo a evitar enviesamento dos resultados.

A significância dos coeficientes de regressão foi avaliada após a estimação dos parâmetros pelo método da máxima verosimilhança. Finalmente, para analisar a qualidade de ajustamento do modelo, recorreu-se aos seguintes indicadores: a) Qui-Quadrado $\left(\chi^{2}\right)$; b) Non-Normed Fit Index (NNFI) e Comparative Fit Index (CFI - deverá ser igual ou superior a 0,95); c) Root Mean-Squared Error of Approximation (RMSEA - não deve ultrapassar 0,06); d) índices de ajustamento local magnitude do Squared Multiple Correlation Coefficient $\left(\mathrm{R}^{2}\right)$, significância do $\mathrm{R}^{2}$, variâncias e covariâncias e quantidade de erro associado (Hu \& Bentler, 1999).

\section{RESULTADOS}

No ajustamento dos modelos analisados - Português e Matemática - para as três fases do estudo (A, B e C), verificaram-se relações comuns aos dois modelos, bem como outras distintas. De modo a facilitar uma interpretação das mesmas, com ênfase nas principais diferenças e semelhanças, os resultados de ambos os modelos serão apresentados de forma integrada nas secções seguintes, que são: ajustamento dos modelos - indicadores do ajustamento final global e local de cada modelo; efeitos intra e interconstructos das $C P C$ - trajetórias de impacto 


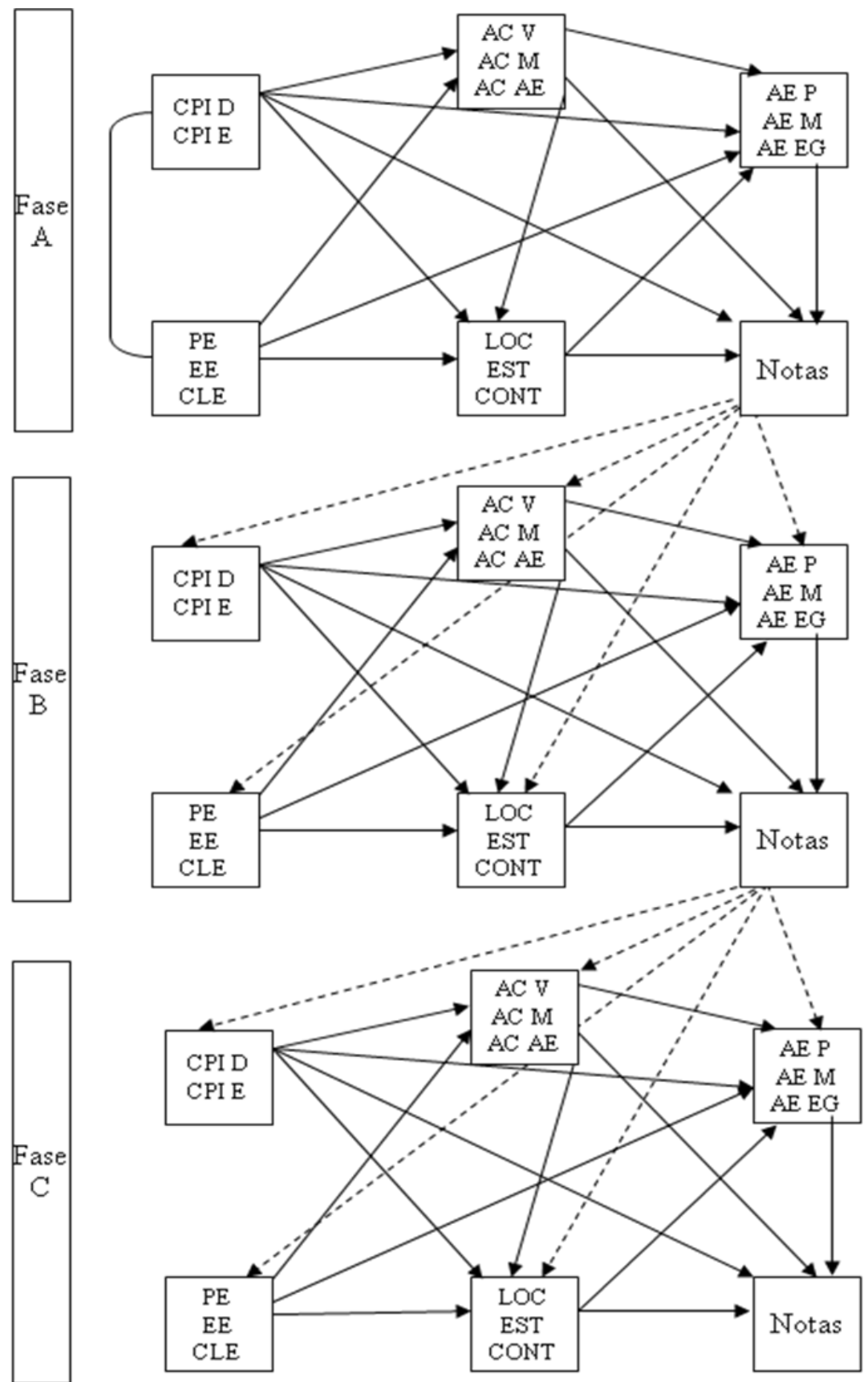

Figura 1. Modelo integrador das concepções pessoais de competência e a sua relação com o rendimento escolar ao longo do ensino secundário (Fases $\mathrm{A}, \mathrm{B}$ e C). $\mathrm{CPI} \mathrm{D}=$ concepções pessoais de inteligência dinâmicas; $\mathrm{CPI} \mathrm{E}=$ concepções pessoais de inteligência estáticas; $\mathrm{PE}=$ percepção emocional; $\mathrm{EE}=$ expressão emocional; $\mathrm{CLE}=$ capacidade para lidar com a emoção; $\mathrm{AC} \mathrm{V}=$ autoconceito verbal; $\mathrm{AC} \mathrm{M}=$ autoconceito Matemática; $\mathrm{AC} \mathrm{AE}=$ autoconceito assuntos escolares; LOC = locus; EST - estabilidade; CONT = controlabilidade; $\mathrm{AE} \mathrm{P}=$ autoeficácia Português; $\mathrm{AE} \mathrm{M}=$ autoeficácia Matemática; AE EG = autoeficácia escolar geral.

das variáveis psicológicas sobre elas próprias ao longo das três fases de estudo (objetivo [d] deste trabalho) e entre as diferentes variáveis dentro de cada fase (objetivo [a]); efeitos dos constructos das CPC sobre o rendimento - trajetórias de impacto das variáveis psicológicas sobre o rendimento dentro de cada fase (objetivo [b]); efeitos do rendimento sobre os constructos das $C P C$ - trajetórias de impacto do rendimento sobre as variáveis psicológicas entre as fases (objetivo [c]).
De forma a evitar alguma repetição na remissão para a visualização dos resultados, é importante informar que os resultados relativos a cada modelo com as trajetórias, ao longo do ensino secundário, podem ser consultados na Figura 2 (rendimento em Português) e na Figura 3 (rendimento em Matemática). Devido à complexidade dos modelos e, por conseguinte, dos resultados, não estão integradas as relações intraconstructos nas Figuras supracitadas, sendo os respectivos valores de $\mathrm{R}^{2}$ apresentados na Tabela 1 . 


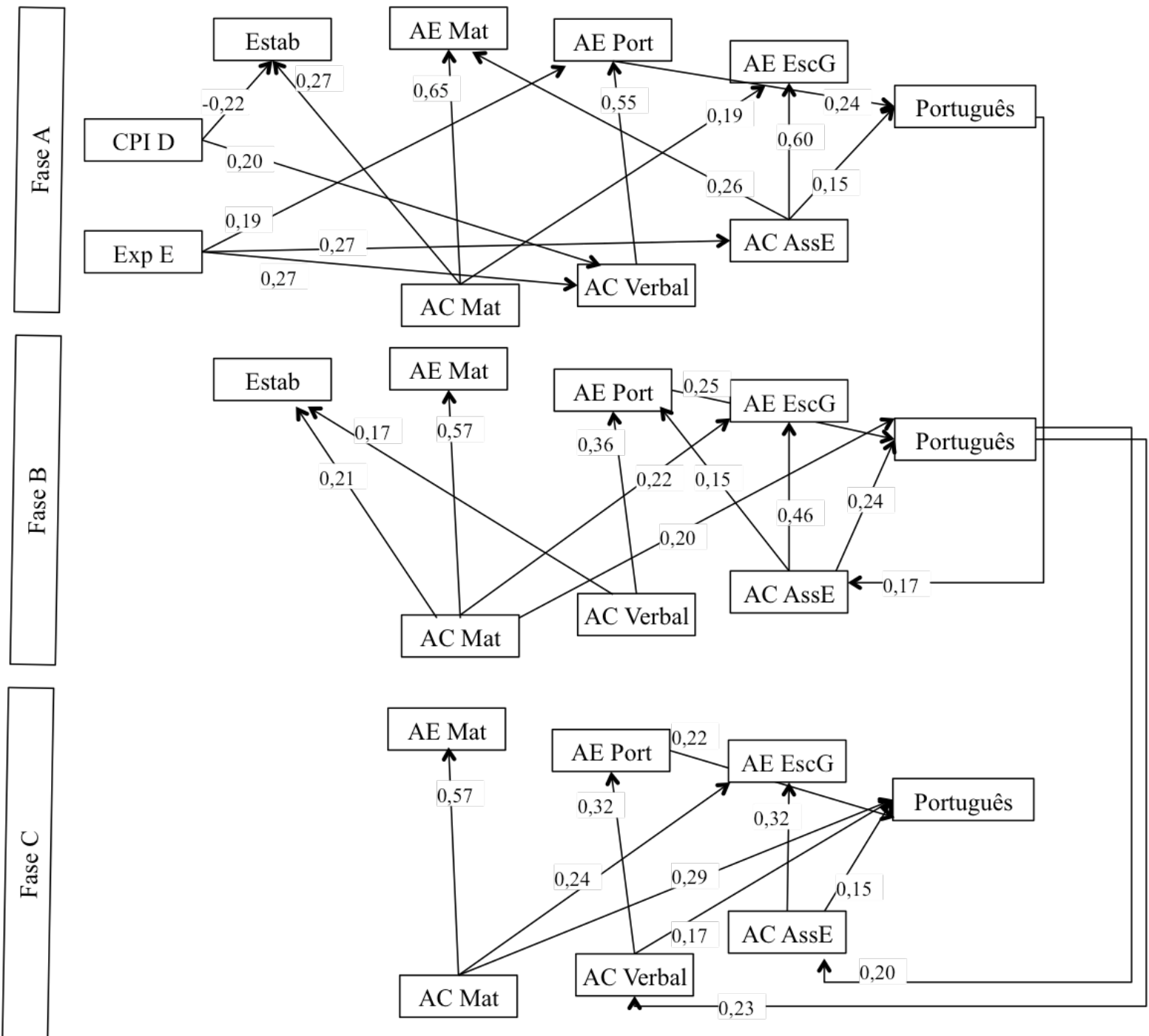

Figura 2. Modelo das relações significativas obtidas no modelo causal de Português ao longo do ensino secundário (Fases A, B e C). CPI D = concepções pessoais de inteligência dinâmicas; Exp E = expressão emocional; $\mathrm{AC}$ - autoconceito; Mat = Matemática; AssE = assuntos escolares; Estab = estabilidade; $\mathrm{AE}=$ autoeficácia; Port $=$ Português; EscG $=$ escolar geral; Português $=$ rendimento de Português.

Tabela 1. Coeficientes de Regressão estandardizados ( $\beta$ ) para as trajetórias intraconstrutos significativas nos modelos de Português e de Matemática

\begin{tabular}{|c|c|c|c|c|c|c|}
\hline \multirow{2}{*}{$\begin{array}{l}\text { Dimensões/ } \\
\text { Fases }\end{array}$} & \multicolumn{3}{|c|}{ Modelo Port } & \multicolumn{3}{|c|}{ Modelo Mat } \\
\hline & $\mathbf{A} \rightarrow \mathbf{B}$ & $\mathrm{B} \rightarrow \mathrm{C}$ & $\mathrm{A} \rightarrow \mathrm{C}$ & $\mathbf{A} \rightarrow \mathbf{B}$ & $\mathrm{B} \rightarrow \mathrm{C}$ & $\mathbf{A} \rightarrow \mathbf{C}$ \\
\hline CPI Dinâmica & & & & 0,58 & 0,57 & 0,16 \\
\hline $\begin{array}{l}\text { Expressão } \\
\text { Emocional }\end{array}$ & & & & 0,54 & & \\
\hline Estabilidade & 0,30 & & & 0,27 & & \\
\hline Locus & & & & 0,27 & 0,15 & \\
\hline Controlabilidade & & & & 0,40 & 0,26 & 0,17 \\
\hline AC Verbal & 0,59 & 0,38 & 0,41 & 0,64 & 0,45 & 0,38 \\
\hline AC Matemática & 0,74 & 0,62 & 0,23 & 0,59 & 0,46 & 0,26 \\
\hline $\begin{array}{l}\text { AC Assuntos } \\
\text { Escolares }\end{array}$ & 0,65 & 0,58 & 0,20 & 0,62 & 0,62 & 0,25 \\
\hline AE Português & 0,39 & 0,38 & 0,11 & 0,42 & 0,42 & 0,23 \\
\hline AE Matemática & 0,32 & 0,21 & 0,20 & 0,34 & 0,18 & 0,16 \\
\hline AE Esc Geral & 0,32 & 0,35 & 0,23 & 0,37 & 0,37 & 0,21 \\
\hline
\end{tabular}

Nota . Port = Português; Mat = Matemática; $\mathrm{AC}=$ autoconceito $; \mathrm{AE}=$ autoeficácia; Esc $\mathrm{G}=$ escolar geral. $\rightarrow$ 


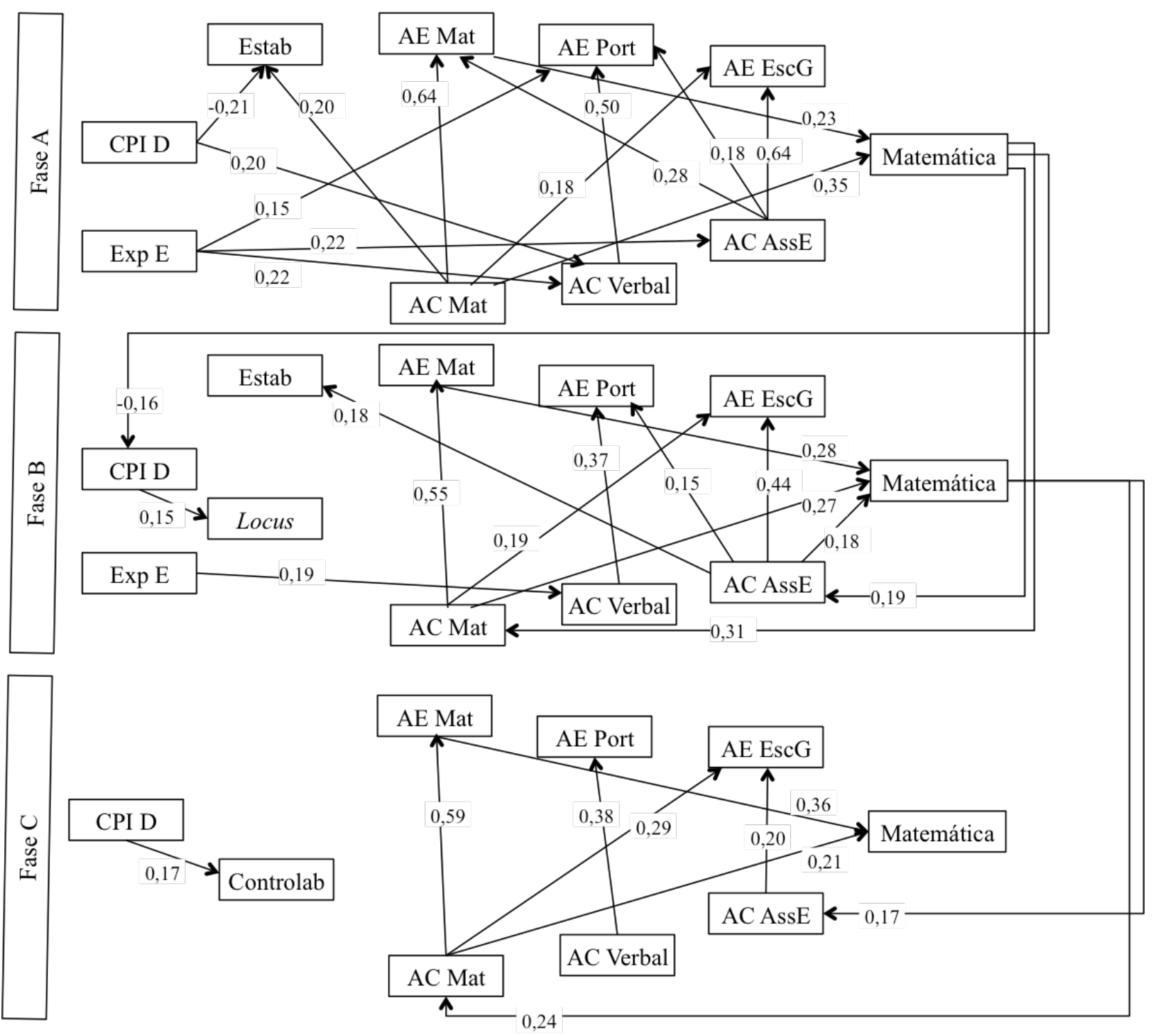

Figura 3. Modelo das relações significativas obtidas no modelo causal de Matemática ao longo do ensino secundário (Fases A, B e C). CPI D = concepções pessoais de inteligência dinâmicas; Exp E = expressão emocional; $\mathrm{AC}=$ autoconceito; Mat =Matemática; AssE = assuntos escolares; Estab = estabilidade; Controlab = controlabilidade; $\mathrm{AE}$ = autoeficácia; Port = Português; EscG -escolar geral; Matemática = rendimento de Matemática.

\section{Ajustamento dos Modelos}

Uma vez que foram ajustados dois modelos diferentes (Português e Matemática) e que cada um passou por várias etapas, de modo a integrar passo a passo as variáveis e respectivas relações intra e interfases $(\mathrm{A}, \mathrm{B}$ e C) descritas na secção da análise dos dados, serão apenas descritos os resultados dos modelos finais, integrando as três fases.

Em relação aos modelos finais ajustados, ambos apresentaram índices globais semelhantes entre si, com alguns indicadores considerados sofríveis, ou seja, um pouco abaixo dos limites considerados satisfatórios, como é possível ver a seguir: (a) modelo de Português - $\chi^{2}=931,2 ; p=0,00$; $g l=423 ; \chi^{2} / g l=2,20 ; \mathrm{NNFI}=0,91 ; \mathrm{CFI}=0,93 ; \mathrm{RMSEA}=0,07$; (b) modelo de Matemática - $\chi^{2}=1107,16 ; p=0,00 ; g l=550$; $\chi^{2} / g l=2,01 ; \mathrm{NNFI}=0,90$; CFI $=0,92$; RMSEA $=0,06$. Uma vez que foram apenas mantidas as relações com valores de significância inferiores a 0,01 e com valores $\beta$ superiores a 0,15 , como referido na análise de dados, os índices de ajustamento local são globalmente aceitáveis, o que verifica que a quantidade de erro associado se situa essencialmente entre 0,01 e 0,05 , e que os valores de $R^{2}$ variam entre 0,15 e 0,74 . Contudo, os valores de $R^{2}$ fortes situam-se essencialmente nas relações intraconstructos, sendo as relações interconstructos geralmente caraterizadas por valores mais fracos, implicando uma análise dos resultados mais parcimoniosa, bem como a necessidade de estudos futuros para permitir uma compreensão mais integrada do assunto. 
Em relação à variância explicada, é importante mostrar que nos dois modelos houve um aumento com a introdução progressiva das variáveis de cada fase. No final, o modelo que explicou maior quantidade de variância foi o de Matemática, já que a porcentagem de $55 \%$ da variabilidade das notas desta disciplina no ensino secundário parece ser explicada pelo modelo final ajustado. Já o modelo de Português explicou $36 \%$ da variabilidade das suas notas ao longo do ciclo de ensino analisado.

\section{Efeitos Intra e Interconstructos das CPC}

No que diz respeito à estrutura final dos modelos analisados, é necessário frisar antes que as dimensões de capacidade para lidar com a emoção, percepção emocional e CPI estática não integraram os modelos por não se relacionarem com nenhuma variável.

No âmbito dos efeitos intraconstructos, como esperado, todas as variáveis geraram um efeito positivo em si mesmas ao longo dos três anos do ensino secundário (da fase $\mathrm{A}$ até as fases B e C), reforçando o seu caráter desenvolvimental e cumulativo. De fato, todas as trajetórias de impacto apresentaram sempre valores $\beta$ superiores a 0,15 e níveis de significância inferiores a 0,01 , exceto a autoeficácia em Português no modelo de Português da fase A para a fase C ( $\beta=0,11, p=0,03$ - Tabela 1$)$.

$\mathrm{Na}$ análise das relações entre as variáveis das $\mathrm{CPC}$, primeiramente deve-se recordar que a CPI foi proposta como dimensão estruturante do modelo (Figura 1). Contudo, em ambos os modelos, as relações significativas mais moderadas se situam essencialmente no $10^{\circ}$ ano, influenciando positivamente o autoconceito verbal e negativamente a estabilidade. Tal resultado sugere que CPI mais dinâmicas estão na origem de percepções de competência verbal mais positivas e de percepções causais mais instáveis ao longo do ano letivo, o que ocorreu de acordo com o esperado (Blackwell et al., 2007; Ferla et al., 2009; Pina Neves \& Faria, 2005). Especificamente, no modelo para o rendimento em Matemática, a CPI dinâmica parece exercer igualmente influência positiva no locus e na controlabilidade no $11^{\circ}$ e $12^{\circ}$ ano, respectivamente. Essas relações, embora fracas, poderão sugerir que CPI mais dinâmicas contribuem para uma maior internalização das causas no $11^{\circ}$ ano e maior percepção de controlo no $12^{\circ}$ ano, embora seja importante verificar este resultado em futuros estudos.

Tal como a CPI, a competência emocional também foi proposta como dimensão estruturante do modelo das CPC (Figura 1). A expressão emocional (EE), única variável emocional a integrar os modelos, parece ter impacto positivo mais moderado no $10^{\circ}$ ano e nos domínios da língua materna e das disciplinas em geral, afastando-se um pouco da previsão de variável estruturante do modelo. Assim, parece influenciar direta e positivamente no autoconceito verbal, em assuntos escolares e na autoeficácia em Português no $10^{\circ}$ ano dos dois modelos analisados. Excepcionalmente, a EE parece também ter alguma influência positiva no autoconceito verbal no $11^{\circ}$ ano do modelo de Matemática.

No que diz respeito às relações de influência das dimensões do autoconceito acadêmico, o modelo das CPC propunha que estas relações determinariam diretamente as dimensões causais e a autoeficácia acadêmica (Figura 1). Grande parte destas relações é corroborada, fazendo com que o autoconceito seja a dimensão com impacto mais moderado noutros constructos e com principal incidência nas dimensões correspondentes da autoeficácia do mesmo domínio acadêmico. Efetivamente, e de forma transversal aos modelos analisados e aos três anos do ensino secundário, verifica-se que as relações entre o autoconceito e a autoeficácia são moderadas no mesmo domínio acadêmico e mais fracas em domínios diferentes: o autoconceito verbal é a variável com maior impacto na autoeficácia em Português, o autoconceito Matemática é o principal determinante da autoeficácia em Matemática e o autoconceito assuntos escolares é o principal preditor da autoeficácia escolar geral, o que vai de encontro ao previsto, embora com valores um pouco menos elevados (Pina Neves \& Faria, 2005). Com menor intensidade, o autoconceito Matemática parece também influenciar positivamente a autoeficácia escolar geral nos dois modelos e nos três anos. Por sua vez, o autoconceito assuntos escolares também parece ter impacto positivo na autoeficácia em Matemática e em Português em diferentes anos de escolaridade nos dois modelos em análise. Finalmente, as três dimensões do autoconceito, como explicado a seguir, também parecem ter alguma influência positiva na estabilidade: no $10^{\circ}$, através do autoconceito Matemática (nos dois modelos), e no $11^{\circ}$ ano, através dessa mesma dimensão, do autoconceito verbal (ambos no modelo de Português) e do autoconceito assuntos escolares (modelo de Matemática). Tal resultado parece sugerir que percepções de competência positivas no $10^{\circ}$ e $11^{\circ}$ anos originam percepções causais mais estáveis ao longo destes anos letivos.

\section{Efeitos dos Constructos das CPC sobre o Rendimento}

O modelo das CPC propunha que o rendimento seria diretamente influenciado pelas CPI, atribuições e dimensões causais, autoconceito e autoeficácia acadêmicos em cada fase (A, B e C - Figura 1). Contudo, o autoconceito e autoeficácia acadêmicos parecem ser os mais relevantes, apresentando efeitos positivos e moderados sobre as notas.

Efetivamente, a nota de Português, bem como o modelo de Português, são influenciados pelas três dimensões do autoconceito acadêmico, considerando que o autoconceito assuntos escolares é significativo nos três anos, o autoconceito Matemática influencia no $11^{\circ}$ e $12^{\circ}$ anos e o autoconceito verbal apenas tem impacto no $12^{\circ}$ ano.

Das dimensões que constituem a autoeficácia acadêmica, 
apenas a autoeficácia em Português é significativa no modelo, sendo, no entanto, a dimensão mais influente na nota de Português.

No modelo de Matemática, as duas dimensões mais afins deste domínio escolar são as que parecem ter maior impacto, isto é, autoconceito e autoeficácia em Matemática, sugerindo que percepções de competência e expectativas positivas no campo da Matemática estão na base de bons resultados nesta disciplina. $\mathrm{O}$ autoconceito assuntos escolares também influencia, embora com menor magnitude, o rendimento em Matemática no $11^{\circ}$ ano.

\section{Efeitos do Rendimento sobre os Constructos das CPC}

O modelo das CPC propunha também influência direta do rendimento do ano anterior nas variáveis psicológicas do ano seguinte, ou seja, a nota obtida no $10^{\circ}$ ano teria impacto nas dimensões psicológicas no $11^{\circ}$ ano e a nota deste ano teria igualmente impacto no $12^{\circ}$ ano (Figura 1). Contudo, nem todas as variáveis demonstraram sofrer impacto direto do rendimento, o qual tem especial efeito nas dimensões do autoconceito.

Efetivamente, no modelo de Português, a relação mais forte se verifica entre a nota e o autoconceito verbal do $11^{\circ}$ para o $12^{\circ}$ ano, sendo também significativa, mas menos forte, a influência da nota sobre o autoconceito assuntos escolares no $11^{\circ}$ e $12^{\circ}$ anos. Novamente estas relações são positivas, indicando que quanto melhor a nota em Português, mais positivo o autoconceito verbal e de assuntos escolares.

Igualmente o modelo anterior, em que a relação mais forte se situou num constructo envolvido ao domínio escolar, a dimensão mais influenciada pela nota em Matemática do ano anterior é o autoconceito Matemática, seguido, mais uma vez, pelo autoconceito assuntos escolares. Estas relações são válidas para o $11^{\circ}$ e $12^{\circ}$ anos e são moderadas a fortes e positivas, sugerindo que boas notas a Matemática darão origem, no ano seguinte, a percepções positivas de competência em Matemática e nas disciplinas em geral. A exceção surge na influência negativa da nota de Matemática sobre a CPI dinâmica no $11^{\circ}$ ano, indicando que boas notas nesta disciplina originam CPI menos dinâmicas (mais estáticas).

\section{DISCUSSÃo}

No presente trabalho, foi analisada a relação entre as variáveis do modelo das $\mathrm{CPC}$, suas relações e o rendimento escolar ao longo do ensino secundário em Português, prevendo a existência de efeitos recíprocos (Marsh et al., 1999) e o impacto das variáveis sobre si mesmas ao longo dos três anos. A seguinte discussão será organizada por constructo, discutindo de forma integrada o papel de cada variável no contexto dos modelos analisados.

Apesar de o modelo das CPC propor as dimensões da CPI como estruturantes do modelo (Figura 1), apenas a CPI dinâmica foi significativa, parecendo refletir maior relevância na relação com outras variáveis do que a CPI estática, embora os valores fossem relativamente fracos e necessitem de estudos posteriores. De fato, apesar de não ter impacto em todas as dimensões consideradas e nem confirmação da hipótese do efeito indireto sobre a autoeficácia, a existência de CPI dinâmicas, ou seja, de crença na possibilidade de desenvolvimento da competência intelectual e numa maior importância dada ao processo de aprendizagem do que aos resultados per se (Dweck, 1996), parece prestar algum contributo para: (a) um autoconceito mais positivo, especialmente na língua materna; (b) maior responsabilização pelos resultados (locus de causalidade mais interno); (c) maior percepção de controlo; (d) percepções causais mais instáveis, refletindo o seu caráter mais dinâmico que permite diferenciar atribuições causais em função do contexto. Estes resultados vão de encontro ao previsto pelo modelo das CPC e por outros estudos na mesma linha (Dweck, 1996; Pina Neves \& Faria, 2005; Yeager \&
Dweck, 2012). Contrariamente ao esperado (Dweck, 1996; Stocker et al., 2011; Yeager \& Dweck, 2012), a CPI dinâmica não teve impacto em nenhum dos rendimentos analisados, reforçando a determinação e estruturação dos constructos psicológicos como mais importante do que diretamente no rendimento objetivo. A relação inversa, no que diz respeito à influência do rendimento sobre a CPI dinâmica, foi verificada para o rendimento de Matemática, porém no sentido negativo, sugerindo que bons resultados nestes domínios acadêmicos poderão dar origem a CPI menos dinâmicas no ano seguinte. Apesar de estes resultados serem fracos e inconsistentes, poder-se-á refletir sobre o seguinte: os $11^{\circ}$ e $12^{\circ}$ anos constituem um período escolar em que os alunos podem apresentar alguma preocupação em relação aos resultados, devido à necessidade de obtenção de elevadas notas para acesso ao ensino superior (universidade). Assim, os alunos com melhores notas em Matemática num ano poderão apresentar no ano seguinte objetivos mais centrados nos resultados e CPI menos dinâmicas, de modo a alcançar os objetivos acadêmicos a que se propuseram (Stocker et al., 2011). Contudo, mais uma vez salientamos que se tratam de hipóteses explicativas destes resultados, necessitando de estudos posteriores de aprofundamento.

Nas dimensões da competência emocional, apenas a EE revelou alguma importância para a estrutura do modelo das CPC. As relações significativas, embora globalmente fracas, corroboram a teoria de Mayer e Salovey (1997), bem como a de Brackett et al. (2011), uma vez que níveis elevados de EE parecem contribuir para concepções de competência 
(autoconceito) e de eficácia mais positivas, essencialmente em domínios escolares gerais e na língua materna. Quanto à relação direta prevista com as atribuições e dimensões causais, não houve confirmação, já que a competência emocional não parece ter impacto relevante na interpretação das causas dos acontecimentos escolares. A respeito do rendimento, os presentes resultados corroboraram os estudos que encontraram uma relação não significativa com a competência emocional (Ahammed et al., 2011; Stocker et al., 2011).

Por sua vez, com valores globalmente moderados, o autoconceito apresentou-se como o constructo mais influente nas outras variáveis, especialmente sobre a autoeficácia e, tal como previsto, sobre dimensões afins no que diz respeito ao domínio escolar (Ferla et al., 2009; Pajares \& Schunk, 2001; Pina Neves \& Faria, 2005). O autoconceito relacionado a assuntos escolares e Matemática parece ser mais transversal no seu impacto interconstructos, enquanto o autoconceito verbal é mais circunscrito à autoeficácia na língua materna e à estabilidade, ao contrário do que se poderia esperar, chamando a atenção para a importância da transversalidade do domínio da Matemática. Assim, a influência do autoconceito sobre a autoeficácia e dimensões causais, considerada no modelo das CPC, foi parcialmente corroborada, contribuindo para a estabilidade das percepções causais e para a construção de expectativas de realização acadêmica positivas. Os efeitos moderados recíprocos entre autoconceito e rendimento acadêmico, previstos no modelo de Marsh et al. (1999) e no modelo das CPC, foram corroborados. Tal como seria de esperar, devido à sua transversalidade acadêmica, o autoconceito assuntos escolares tende a influenciar e ser influenciado pelos dois tipos de rendimento acadêmico, isto é, o autoconceito Matemática influencia e é influenciado predominantemente pelo rendimento de Matemática, embora tenha igualmente algum impacto na nota a Português; e o autoconceito verbal influencia e é influenciado pelo rendimento de Português na Fase $\mathrm{C}$, ficando de novo demonstrada a importância da dimensão de Matemática do autoconceito. Globalmente, fica mais uma vez comprovada a relação causal recíproca e positiva entre o autoconceito e o rendimento escolar, sendo que quanto mais elevado o autoconceito, melhores as notas e vice-versa.
Contrariando o esperado com base em Weiner (1985), as atribuições e dimensões causais não parecem contribuir de forma relevante para a formação de expectativas no contexto escolar (autoeficácia), sendo apenas alvo de influência de outras variáveis, como do autoconceito e CPI dinâmica. Também não se identifica contribuição para o rendimento acadêmico de Português e de Matemática, sendo as variáveis com menor peso no modelo das CPC.

O impacto das dimensões da autoeficácia previsto pelo modelo das CPC sobre o rendimento acadêmico foi confirmado, uma vez que influenciou moderadamente o rendimento, principalmente nos domínios afins, o que refletiu seu caráter microanalítico (Bandura, 1977; Caprara et al., 2008; Pina Neves \& Faria, 2005). Já o efeito recíproco, isto é, de influência do rendimento sobre a autoeficácia, não se verifica. Tal acontecimento pode ter base no caráter microanalítico e contextual da autoeficácia (Bandura, 1977), uma vez que foi analisado o impacto do rendimento do ano anterior sobre as expectativas do ano seguinte. Possivelmente, variáveis mais próximas contextual e temporalmente serão mais relevantes na construção de expectativas de resultados escolares, como demonstrou ser o autoconceito acadêmico.

A corroboração do impacto positivo das variáveis das CPC sobre si próprias ao longo do ensino secundário, também prevista no modelo proposto e com base nas recomendações teóricas e metodológicas de Marsh et al. (1999), parece reforçar a importância da sua promoção no contexto acadêmico desde cedo, uma vez que as autopercepções de um ano determinarão as mesmas do ano seguinte e nos posteriores, como foi observado.

Pode-se, então, concluir que o modelo das CPC proposto encontra fundamentos empíricos para grande parte das suas relações, sejam as relações intra e interconstructos, sejam as relações recíprocas com o rendimento. Contudo, é importante considerar que a intensidade das relações foi globalmente de fraca a moderada e as conclusões deste estudo devem se abster de generalizações diretas a outros contextos. Assim, enfatiza-se a importância da realização de mais estudos longitudinais que possam analisar, de forma sistemática, trajetórias de impacto de variáveis psicológicas e do rendimento ao longo do tempo.

\section{CONSIDERAÇÕES FINAIS}

Os resultados do presente trabalho parecem reforçar a relevância do modelo integrador das CPC das relações entre as suas componentes e da reciprocidade relacional destas com o rendimento acadêmico. Assim, as variáveis motivacionais parecem assumir novamente um papel central para a competência e para o sucesso acadêmico, apelando à necessidade de promoção de CPC positivas nos alunos, não só por parte das comunidades escolares, isto é, das escolas e dos professores, como também por parte das famílias e dos encarregados de educação.
Este estudo, tal como muitos nesse domínio, apresenta algumas limitações que, sendo devidamente identificadas, poderão ser evitadas em estudos futuros desta natureza, sendo igualmente uma oportunidade para refletir acerca de outras possibilidades e obter pistas para investigações futuras.

Em relação à amostra, primeiramente, apesar dela ser composta por 433 alunos, em cada modelo foi necessário retirar os respectivos casos omissos, ficando uma amostra mais reduzida. Por outro lado, poderia ser interessante comparar o modelo das CPC em alunos de diferentes 
cursos, procurando perceber o perfil motivacional do aluno de Ciências e Tecnologias e de Línguas e Humanidades, por exemplo, pois além de cada curso atrair alunos com diferentes características, as disciplinas e os objetivos de cada curso também podem interferir na construção das teorias pessoais de competência. Outra possibilidade seria alargar este estudo longitudinal do ensino secundário para o ensino superior, fazendo um follow-up dos alunos, percebendo se houve alterações no seu perfil motivacional de acordo com o seu percurso acadêmico (entrada na faculdade, curso de primeira opção, etc.) ou mesmo ciclos de escolaridade anteriores, comparando os modelos e respectivas relações. Nestas situações, será importante adaptar o QCCP aos anos de escolaridade em questão, pois alguns dos itens foram especificamente construídos para o ensino secundário. Decorrendo deste aspeto, outra limitação refere-se ao tipo de avaliação dos constructos, pois se uns dizem respeito a situações de realização e/ou escolares específicas, como a EAEA, outros são mais generalistas, como o QCE. Especificamente em relação ao QCE, seria pertinente adequá-lo ao contexto acadêmico, abordando, por exemplo, a capacidade de os alunos gerirem emocionalmente situações de avaliação, sucesso, fracasso, competitividade e pressão para a obtenção de resultados, entre outros. O QADC também poderia se beneficiar de uma reformulação dos seus itens através da diferenciação de situações de sucesso e de fracasso, permitindo uma interpretação dos resultados mais objetiva e contextualizada.

Metodologicamente, sugere-se a realização de modelos causais mais parcimoniosos, de forma a ser possível integrar variáveis latentes e utilizar metodologias mais adequadas para medidas repetidas através de modelos de crescimento latente (AMOS/HLM). Com recurso a análises multinível (HLM), seria igualmente interessante analisar, além da evolução intraindividual, diferenças interindividuais, como o sexo, nível socioeconômico, planos futuros (vontade de continuar a estudar/ trabalhar) e intergrupos (curso, turma, escola). Tais recomendações encontram fundamento em várias teorias, como a de Bandura (1998) e a de Mayo (1945), que enfatizam o fato de o comportamento humano, as concepções pessoais de competência, e até a própria produtividade/ eficácia/rendimento terem também origem no quadro de relações humanas e interações sociais (Sarachek, 1968). Na verdade, o comportamento individual está dependente do comportamento coletivo e das normas do grupo de pertença do indivíduo, que funcionam como "sociostructural influences" (Bandura, 1998, p. 51). Tais influências concretizam-se através da capacidade de integração social do indivíduo e das percepções e crenças desenvolvidas dentro do respectivo grupo acerca da possibilidade de sucesso coletivo (eficácia coletiva, cf. Bandura, 1998), contribuindo significativamente para a forma como o indivíduo percepciona a si mesmo e à tarefa e para o resultado final. Assim, recomenda-se, para futuras investigações, a inclusão de variáveis que tomem em consideração aspetos coletivos, como a eficácia coletiva, permitindo a análise da relação entre variáveis "individuais" e "coletivas" e o respectivo impacto no rendimento acadêmico.

Ao mesmo tempo que se confirma a exequibilidade e importância do modelo das CPC proposto para o ensino secundário, fica igualmente clara a necessidade de novas investigações que tenham em consideração as limitações e as sugestões apontadas. Pode-se recomendar, para futuros estudos, essencialmente a realização de estudos longitudinais como este, que permitem analisar a evolução intraindividual das variáveis ao longo do tempo. Também é importante que seja (re)testado o modelo das CPC, os resultados obtidos e eventualmente estudos longitudinais-sequenciais que comparem resultados longitudinais e transversais.

\section{REFERÊNCIAS}

Ahammed, S., Abdullah, A., \& Hassane, S. (2011). The role of emotional intelligence in the academic success of UAE university students. International Education, 41(1), 7-25.

Bandura, A. (1977). Self-efficacy: Toward a unifying theory of behavioral change. Psychological Review, 84(2), 191-215.

Bandura, A. (1998). Personal and collective efficacy in human adaptation and change. In J. G. Adair, D. Belanger, \& K. L. Dion (Eds.), Advances in psychological science, Volume 1: Social, Personal and Cultural Aspects (pp. 51-71). Hove, UK: Psychology Press.

Berger, C., Alcalay, L., Torretti, A., \& Milicic, N. (2011). Socio-emotional well-being and academic achievement: Evidence from a multilevel approach. Psicologia: Reflexão e Crítica, 24(2), 344-351. https://doi.org/10.1590/S010279722011000200016

Blackwell, L. S., Trzesniewski, K., \& Dweck, C. S. (2007). Implicit theories of intelligence predict achievement across an adolescent transition: A longitudinal study and an intervention. Child Development, 78(1), 246-263. https://doi.org/10.1111 j.1467-8624.2007.00995

Brackett, M. A., Rivers, S., \& Salovey, P. (2011). Emotional intelligence: Implications for personal, social, academic, and workplace success. Social and Personality Psychology Compass, 5(1), 88-103. https://doi.org/10.1111/j.1751-9004.2010.00334

Byrne, B. M. (1984). The general/academic self-concept nomological network: A review of construct validation research. Review of Educational Research, 54, 427-456. https:// doi.org/10.3102/00346543054003427

Caprara, G. V., Fida, R., Vecchione, M., Bove, G., Vecchio, G. M., Barbaranelli, C., \& Bandura, A. (2008). Longitudinal analysis of the role of perceived self-efficacy for self-regulated learning in academic continuance and achievement. Journal of Educational Psychology, 100(3), 525-534. https://doi. org/10.1037/0022-0663.100.3.525

Dweck, C. S. (1996). Implicit theories as organizers of goals and behavior. In P. Gollwitzer \& J. Bargh (Eds.), The Psychology of Action: Linking Cognition and Motivation to Behavior (pp. 69-90). New York: The guildford press.

Faria, L. (2006). Escala de concepções pessoais de inteligência (ECPI): Novos estudos. In M. Gonçalves, M. Simões, L. Almeida, \& C. Machado (Coords.), Avaliação Psicológica. Instrumentos Validados para a População Portuguesa (pp. 121-133). Coimbra: Quarteto Editora. 
Faria, L., \& Fontaine, A. M. (1992). Estudo de adaptação do Self-Description Questionnaire III (SDQ III) a estudantes universitários portugueses. Psychologica, 8, 41-49.

Faria, L., \& Lima Santos, N. (2011). Questionário de competência emocional (QCE). In C. Machado, M. Gonçalves, L. Almeida, \& M. Simões (Eds.), Instrumentos e Contextos de Avaliação Psicológica (Vol. 1, pp. 67-80). Coimbra: Edições Almedina, SA.

Ferla, J., Valcke, M., \& Cai, Y. (2009). Academic self-efficacy and academic self-concept: Reconsidering structural relationships. Learning and Individual Differences, 19, 499-505. https://doi. org/10.1016/j.lindif.2009.05.004

Ferreira, M. E., Assmar, E., Omar, A., Delgado, H., González, A., Silva, J., \& Cisne, M. C. (2002). Atribuição de causalidade ao sucesso e fracasso escolar: Um estudo transcultural BrasilArgentina-México. Psicologia: Reflexão e Crítica, 15(3), 515-527.

Gonzaga, A., \& Monteiro, J. (2011). Inteligência emocional no Brasil: Um panorama da pesquisa científica. Psicologia: Teoria e Pesquisa, 27(2), 225-232. https://doi.org/10.1590/ S0102-37722011000200013

Hu, L., \& Bentler, P. M. (1999). Cutoff criteria for Fit Indexes in covariance structure analysis: Conventional criteria versus new alternatives. Structural Equation Modeling, 6(1), 1-55. https:// doi.org/10.1080/10705519909540118

Maroco, J. (2010). Análise de equações estruturais: Fundamentos teóricos, software \& aplicações. Lisboa: Report Number.

Marsh, H. W., Byrne, B. M., \& Yeung, A. S. (1999). Causal ordering of academic self-concept and achievement: Reanalysis of a pioneering study and revised recommendations. Educational Psychologist, 34, 154-157. https://doi. org/10.3102/00028312039003727

Marsh, H. W. \& Martin, A. (2011). Academic self-concept and academic achievement: Relations and causal ordering. British Journal of Educational Psychology, 81, 59-77. https://doi. org/10.1348/000709910X503501

Mayer, J. D., \& Salovey, P. (1997). What is emotional intelligence? In P. Salovey \& D. Sluyter (Eds.), Emotional development and emotional intelligence: Educational implications (pp. 3-31). New York: Basic Books.

Mayo, E. (1945). The human problems of an industrial civilization. Boston: Harvard University.

Oliveira, M., \& Soares A. (2011). Auto-eficácia, raciocínio verbal e desempenho escolar em estudantes. Psicologia: Teoria e Pesquisa, 27(1), 33-39. https://doi.org/10.1590/S010237722011000100005

Paiva, M. O., \& Lourenço, A. (2011). Rendimento acadêmico: Influência do autoconceito e do ambiente de sala de aula. Psicologia: Teoria e Pesquisa, 27(4), 393-402. https://doi. org/10.1590/S0102-37722011000400002

Pajares, F., \& Schunk, D. H. (2001). Self-beliefs and school success: Self-efficacy, self-concept, and school achievement. In R. Riding \& S. Rayner (Eds.), Perception (pp. 239-266). London: Ablex Publishing.

Petrides, K. V., Frederickson, N., \& Furnham, A. (2011). The role of trait emotional intelligence in academic performance and deviant behavior at school. Personality and Individual Differences, 36, 277-293. https://doi.org/10.1016/S01918869(03)00084-9
Pina Neves, S., \& Faria, L. (2005). Concepções pessoais de competência: Da integração conceptual à intervenção psicopedagógica. Psicologia, 18(2), 101-128.

Pina Neves, S., \& Faria, L. (2007). Autoeficácia académica e atribuições causais em Português e Matemática. Análise Psicológica, 25(4), 635-652.

Sarachek, B. (1968). Elton Mayo's social psychology and human relations. The Academy of Management Journal, 11(2), 189197. https://doi.org/10.2307/255256

Stocker, J., Pina Neves, S., \& Faria, L. (2011). Competência percebida e realização escolar: Que relações em alunos do ensino secundário? Psychologica, 52(1), 427-446.

Stocker, J., \& Faria, L. (2012). Competência percebida no ensino secundário: Do conceito à avaliação através de um questionário compósito. Psicologia, 26(2), 113-140.

Weiner, B. (1985). An attributional theory of achievement motivation and emotion. Psychological Review, 92(4), 548-573. https://doi.org/10.1037/0033-295X.92.4.548

Yeager, D., \& Dweck, C. S. (2012). Mindsets that promote resilience: When students believe that personal characteristics can be developed. Educational Psychologist, 47(4), 302-314. https://doi.org/10.1080/00461520.2012.722805 